Conclusion AFB is independently associated with increased indices of P-sel and D-dimer which indicate platelet activation and thrombosis respectively.

\section{THROMBOEMBOLIC RISK STRATIFICATION, ANTI- THROMBOTIC AND ANTICOAGULATION USE FOR PATIENTS WITH ATRIAL FIBRILLATION, A CLINICAL AUDIT}

doi:10.1136/heartjnl-2011-300198.147

R A Veasey, R Kulanthaivelu, P Patel, D W Harrington. Kent and Sussex Hospital, Tunbridge Wells, UK

Introduction Atrial fibrillation (AF) is the most prevalent arrhythmia in primary and secondary healthcare settings. Thromboembolic (TE) risk assessment and initiation of anti-thrombotic or anticoagulation (AT/AC) therapy, according to level of risk, is recommended in both national and international guidelines. NICE guidance stratifies patients with AF in to low, moderate or high risk categories and recommends "aspirin", "aspirin or warfarin" or "warfarin" therapy respectively. ACC/ESC guidance endorses use of the $\mathrm{CHADS}_{2}$ scoring system and for scores of 0,1 , or $\geq 2$ recommends "aspirin", "aspirin or warfarin" or "warfarin" therapy respectively. In addition, it is recommended that AF episode frequency or subtype (paroxysmal (PAF), persistent (PersAF) or chronic (CAF)) does not influence TE risk assessment. We audited UK cardiologists and general practitioners (GPs) to assess adherence to these guidelines.

Methods We designed an audit questionnaire assessing: (1) use of risk stratification tools, (2) choice of AT/AC for increasing levels of risk, and (3) choice of therapy for a number of hypothetical patients with variable TE risk and variable AF subtype. The questionnaire was distributed by electronic or postal mail to 1176 cardiologists and 621 randomly selected GPs.

Results In total, 421 responses were received (306 cardiologists, 115 GPs). Overall, $91.4 \%$ of responders reported use of TE risk stratification tools (97.1\% cardiologists, $76.5 \%$ GPs, $\mathrm{p}<0.001)$. NICE risk assessment is used by $26.6 \%$ of responders $(24.5 \%$ cardiologists, $32.2 \%$ GPs, $\mathrm{p}=0.14$ ), $\mathrm{CHADS}_{2}$ by $79.3 \%$ (90.2\% cardiologists, $50.0 \%$ GPs, $p<0.001)$. The frequency of reported use of AT/AC for each risk level of the NICE assessment and $\mathrm{CHADS}_{2}$ score are shown in Abstract 147 tables 1 and 2 respectively. Type of AF (PAF/PersAF/ $\mathrm{CAF}$ ) reportedly influences the use of $\mathrm{AT} / \mathrm{AC}$ for $34.3 \%$ or responders (24.2\% cardiologists, 46.3\% GPs, $\mathrm{p}=0.001)$. Abstract 147 figure 1 demonstrates AT/AC usage for each of the following hypothetical patients: 1 . 61 year old, hypertension, PAF episodes twice a year lasting 1-2 h (NICE risk: $\bmod , \mathrm{CHADS}_{2}$ score 1/6). 2. 43 year old, diabetes, PAF episodes weekly lasting 10-12 h (NICE risk: mod, $\mathrm{CHADS}_{2}$ score 1/6). 3. 53 year old, hypertension, CAF (NICE risk: mod, $\mathrm{CHADS}_{2}$ score 1/6). 4.78 year old, no other risk factors, CAF (NICE risk: mod, $\mathrm{CHADS}_{2}$ score 1/6). 5. 76 year old, hypertension, diabetes, PAF episodes 3-4 times per year lasting $<1$ hour (NICE risk: high, $\mathrm{CHADS}_{2}$ score 3/6). 6. 77 year old, hypertension, diabetes, PAF episodes occurring weekly and lasting several hours (NICE risk: high, $\mathrm{CHADS}_{2}$ score 3/6). 7. 80 year old, previous TIA, CAF (NICE risk: high, $\mathrm{CHADS}_{2}$ score 3/6).

Abstract 147 Table 1

\begin{tabular}{lcccc}
\hline NICE Risk & None (\%) & Aspirin (\%) & Aspirin or Warfarin (\%) & Warfarin (\%) \\
\hline Low & 16.7 & 78.3 & 2.9 & 2.1 \\
Moderate & 0.6 & 3.5 & 66.9 & 28.7 \\
High & 0.0 & 0.0 & 4.4 & 95.6 \\
\hline
\end{tabular}

Abstract 147 Table 2

\begin{tabular}{lcccc}
\hline CHADS2 Score & None $(\%)$ & Aspirin (\%) & Aspirin or Warfarin (\%) & Warfarin (\%) \\
\hline Zero & 27.0 & 70.3 & 1.3 & 1.3 \\
One & 4.7 & 45.1 & 43.2 & 7.0 \\
Two & 0.0 & 7.8 & 32.2 & 60.0 \\
Three & 0.0 & 0.8 & 11.5 & 87.7 \\
Four & 0.0 & 0.0 & 5.3 & 94.7 \\
Five & 0.0 & 0.0 & 2.9 & 97.1 \\
Six & 0.0 & 0.0 & 2.9 & 97.1 \\
\hline
\end{tabular}

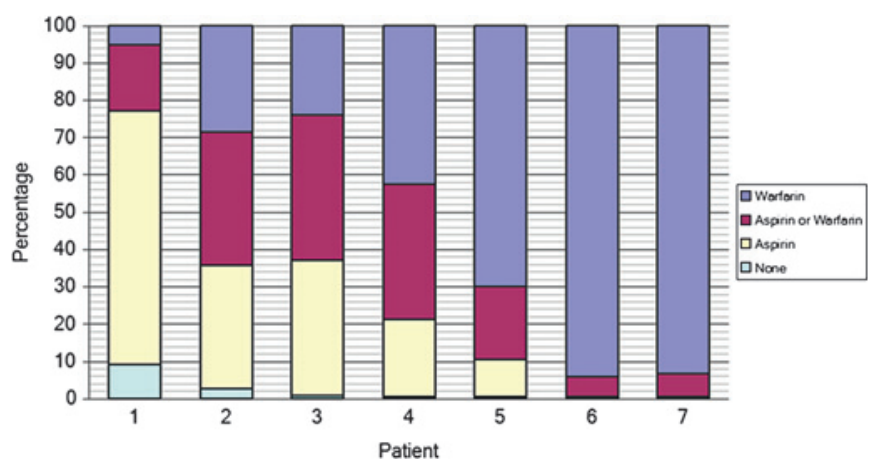

Abstract 147 Figure 1

Conclusions TE risk stratification tools are reportedly widely used in UK clinical practice. AT/AC use for NICE and $\mathrm{CHADS}_{2}$ risk levels are mostly appropriate, although warfarin is under recommended for patients with a $\mathrm{CHADS}_{2}$ score of $2 / 6$. In addition, the use of AT/ $\mathrm{AC}$ is influenced, inappropriately, by AF episode frequency and subtype.

\section{THE ASSESSMENT OF TRANSIENT LOSS OF CONSCIOUSNESS: WE'RE STILL NOT ASKING THE RIGHT QUESTIONS}

doi:10.1136/heartjnl-2011-300198.148

A E Bewick, A Gasson, L Ala, R A Bleasdale. Royal Glamorgan Hospital, Cardiff, UK

Accurately diagnosing patients with TLOC can be achieved in most cases with a detailed clinical history. We set out to assess how patients were assessed in the setting of a district general hospital (DGH) with 570 beds, receiving an unselected intake via general practice and an A\&E. Using the ESC guidelines of 2009 we generated a 22-question study proforma for a retrospective review of the medical records. We identified 322 cases for possible inclusion over a 4 month period. 26 of the case notes were not available to analyse, 8 had insufficient details to identify the relevant patient. Therefore in total 288 notes were reviewed. Inclusion required the TLOC to be complete, of rapid onset and short duration with spontaneous complete recovery. A further 123 patients were therefore excluded. This left 165 data sets (58\% male). The age distribution was a typical bimodal distribution with $16 \%$ between 10 and 29 years of age and $48 \%$ over the age of 70 years. $73 \%$ were assessed in A\&E, $18 \%$ were assessed in the Acute Medical Unit (AMU) and $7 \%$ were assessed in rapid access ambulatory clinics. Only $4 \%$ of the initial assessments were undertaken by consultants, $12 \%$ by a Specialist Registrar (SpR), 21\% by a year 1 foundation program (FP1) doctor and the majority was assessed by FP 2 or core medical trainees (CMT). Key diagnostic elements of the history are still being neglected. For example, the symptoms at the onset of the TLOC were documented in only $58 \%$ of cases; the recovery symptom 